\title{
Manejo periodontal y protésico en sonrisa gingival
}

\author{
Periodontal and prosthetic ganagement of gummy smile for aesthetic purpose
}

\author{
Richard Williams Machaca Uchasara \\ (1) https://orcid.org/0000-0001-9564-2572
}

\section{Resumen}

Los tratamientos de la sonrisa gingival tienen como finalidad reducir la exposición de las encías al sonreír. La causa se debe a uno o varios factores; por tanto, un adecuado diagnóstico es primordial para conseguir el mejor resultado. En este artículo, se reporta un caso clínico que tiene como objetivo presentar el manejo estético periodontal y protésico de un caso de sonrisa gingival con presencia de diastemas y alteraciones en forma. Las herramientas utilizadas para la predictibilidad de la sonrisa fueron un diseño digital y mock up. Los resultados obtenidos fueron estéticos, funcionales y satisfactorios para el paciente.

Palabras clave: alargamiento de corona, espacio biológico, sonrisa gingival

\begin{abstract}
Gummy smile treatments are intended to reduce the exposure of the gums when smiling. The cause is one or more factors; therefore, a proper diagnosis is essential to achieve the best result. In this article, a clinical case is reported that aims to present the periodontal and prosthetic aesthetic management of a case of gummy smile with the presence of diastemas and alterations in shape. The tools used for the predictability of the smile were a digital design and mock up. The results obtained were aesthetic, functional and satisfactory for the patient.
\end{abstract}

Keywords: crown lengthening, biological space, gummy smile

\section{Introducción}

En la actualidad las expectativas estéticas dentales han aumentado, sobre todo en pacientes con sonrisa gingival que representan el $10 \%$ de la población entre las edades de 20 y 30 años y son más frecuente en mujeres. Tjan y et al. ${ }^{1}$ establecieron las directrices de la sonrisa en la década de 1960. Los autores clasificaron la sonrisa en tres categorías: sonrisa alta, media y baja dependiendo de la exposición del margen cervical mediofacial de la corona clínica en relación con el borde inferior del labio superior. Una sonrisa será considerada estéticas cuando los dientes, los labios y la gingival guardan armonía entre ellos. ${ }^{2}$

Las causas de la sonrisa gingival pueden ser: exceso vertical del maxilar, la hipermovilidad del labio superior, labio corto y una alteración de la erupción pasiva o activa. ${ }^{3}$

Diferentes tratamientos se han planteado de acuerdo al causal de la sonrisa gingival. Así ante un exceso vertical del maxilar puede ser tratada mediante la cirugía 
ortognática o un tratamiento de ortodoncia con microtornillos; la hipermovilidad del labio superior puede tratarse con una reposición o temporalmente con toxina botulínica; la alteración en la erupción pasiva o activa produce dientes cortos que se trata con cirugías de alargamiento de corona, aumentando la corona clínica con la reducción del ancho gingival. ${ }^{4,5}$

La sonrisa gingival de causa múltiple se resuelve con el uso de diferentes técnicas combinadas para conseguir los resultados deseados. ${ }^{6,7}$

El diseño digital de sonrisa es una herramienta útil para el diagnóstico que nos permitirá evaluar el tamaño y forma dentaria, así como el contorno gingival estético, teniendo como referencia proporciones y plano faciales de manera que pueda resultar en una armonía entre los labios, contorno gingival y dientes. ${ }^{8,9}$

En la actualidad, los laminados cerámicos son versátiles de trabajar en grosores de 0,3 a 0,5 mm. En la mayoría de los casos, estos laminados requieren de una preparación mínima conservando el $95 \%$ del esmalte. ${ }^{10,11,12}$ Estos laminados nos permiten corregir diastemas, color, forma y posición dentaria. Los estudios clínicos han demostrado el alto porcentaje de éxito al cabo de 10 años de control. ${ }^{13}$

\section{Reporte de caso clínico}

Paciente de sexo femenino de 35 años, quien manifestó su incomodidad estética por presentar una exagerada exposición gingival, formas dentarias y diastemas en el interrogatorio del motivo de consulta. Se realizaron diversos exámenes; al examen extraoral, no revelo hallazgos significativos; al examen intraoral, notamos tratamientos con restauraciones de composite en piezas $2.5,4.6$ y 4.7. Además, se realizaron examen radiográfico, periodontal y estudio de modelos.

\section{Diagnóstico}

i. Diagnóstico sistémico: ASAI

ii. Diagnóstico estomatológico general:

- Caries pieza 1.6, 2.6

- Restauración defectuosa pza 2.5

- Bruxismo excéntrico

iii. Diagnóstico estomatológico periodontal: clasificación de las condiciones y enfermedades periodontales y periimplantarias

iv. Otras condiciones que afectan al periodonto

- Factores relacionados con los dientes y las prótesis

- Factor anatómico dental: pza 1.2, 1.1, 2.1, 2.2

- Erupción pasiva alterada: pza 1.2, 1.1, 2.1,2.2.

\section{Plan de tratamiento}

Fase I

- Educación y motivación respecto a los hábitos de higiene oral

- Eliminación de placa dental

Fase II

- Alargamiento de corona anterosuperior

- Carillas de porcelana sector anterosuperior (3 meses post quirúrgico)

- Férula de relajación y protección

Fase III: Control y motivación

En el presente caso la sonrisa gingival tiene un origen dentogingival a causa de una erupción alterada. Para ese caso, utilizamos como herramienta de planificación el diseño digital de sonrisa. El encerado, guiado por el diseño digital, es transferido mediante el mock up. Con la conformidad manifestada por el paciente de las nuevas proporciones dentales, realizamos la verificación fisiológica: funcional y fonética para el alta del encerado (Figura1). 


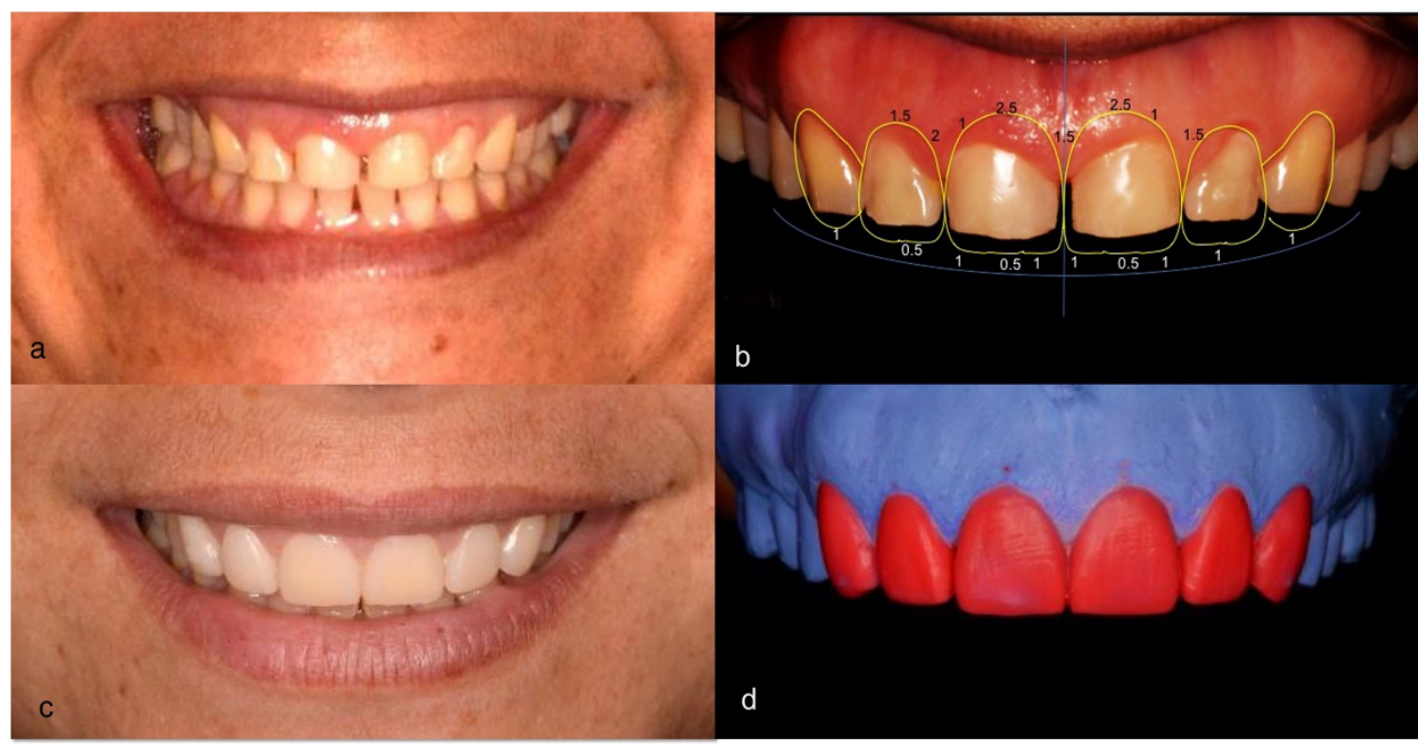

Figura 1. a) Preoperatorio; b) Diseño digital de sonrisa; c) mock up; d) encerado

El mock up se tomó como guía quirúrgica para el alargamiento de corona. Se realizó la incisión con una hoja de bisturí 15c y se retiró el collarín de encía incidida.

Por ser un biotipo periodontal grueso, ejecutamos un levantamiento a espesor total con un remodelado óseo con fresas diamantadas complementadas con cinceles. En consecuencia, el nuevo margen gingival tuvo una distancia de $3 \mathrm{~mm}$ al óseo. Luego, suturamos con un hilo de monofilamento de 5 ceros, los cuales fueron retirados al cabo de 10 días posoperatorio (Figura 2).

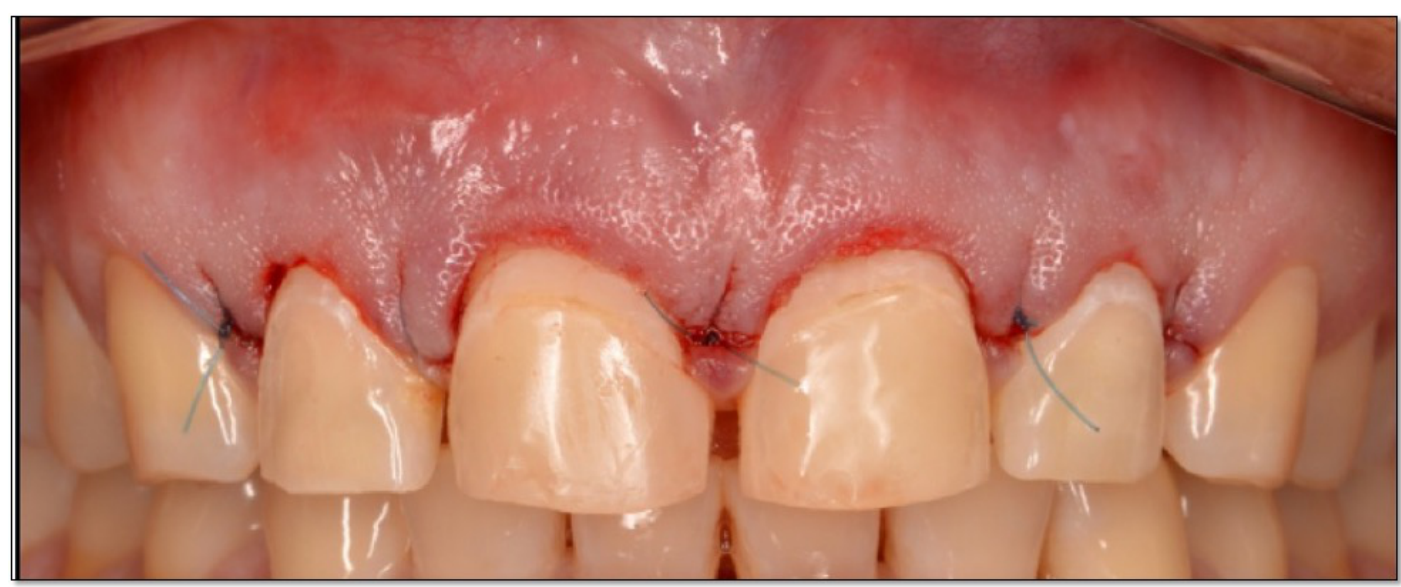

Figura 2.- Posquirúrgico inmediato

Después de 6 meses, se continuó con las preparaciones dentales mediante el uso del mock up y verificando el espacio requerido con llaves de silicona. En este caso clínico, se hizo la preparación para carillas de Di silicato de Litio de $0,5 \mathrm{~mm}$ de grosor, el cual no necesito mayor desgaste porque el paciente ya presentaba perdida de estructura dentaria y diastemas. Por último, cementamos las carillas de porcelana certificando una oclusión orgánica. 


\section{Evolución del caso}

Paciente fue controlado a los 18 meses verificando la estabilidad del contorno gingival y la correcta integracion de las carillas (Figura 3).

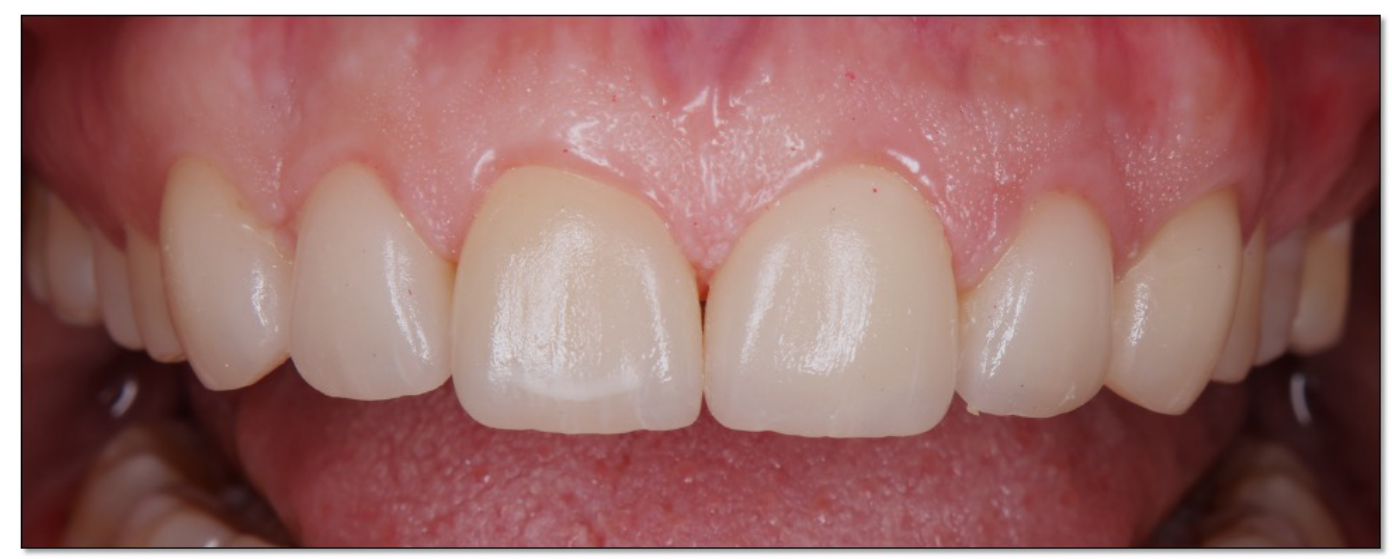

Figura 3. Control a los 18 meses.

\section{Discusión}

La excesiva exposición gingival es una preocupación estética para muchos pacientes y tratarlos un gran desafío. El clínico debe determinar la causa uni o multifactorial a fin de formular un plan de tratamiento clínicamente predecible. ${ }^{14} \mathrm{Se}$ puede realizar un diagnóstico correcto apartir de un examen adecuado, tales como simetría y proporciones faciales, longitud del labio superior y visualización de los dientes en reposo, cantidad de exposición gingival en reposo, durante el habla, sonrisa y la risa. Ya que el plan de tratamiento dependerá de la causa del problema. En este caso, se determinó una causa de origen dentogingival. Seguidamente, se visualizó el resultado estético final con ayuda de un diseño digital de sonrisa.

Riberio et al. ${ }^{15}$ compararon resultados clínicos de la técnica de colgajo a espesor total y sin colgajo para el alargamiento de corona en 28 pacientes a boca dividida. Los autores concluyeron que ambas técnicas dan resultados clínicos estables y similares. Arora et al., ${ }^{16}$ en un ensayo clínico a 64 pacientes, evaluaron las alteraciones de los niveles de tejido periodontal después del alargamiento de corona, durante un periodo de curación de 6 meses. Los autores observaron que la recidiva es mayor en los biotipos periodontales gruesos. En este caso clinico, se realizó un colgajo a espesor total de manera que podamos garantizar el correcto restablecimiento de los $3 \mathrm{~mm}$ de espacio biológico, ademas, se esperó 6 meses para evaluar alguna posible recidiva, ya que se trata de un biotipo periodontal grueso.

Para que el tratamiento restaurador pueda ser predecible, debemos tener estabilidad de los parametros oclusales. Esto porque la articulación temporomandibular se mantendrá saludable si los dientes y estructuras de soporte están en condicion adecuada ${ }^{17}$. En este caso clínico, se evaluó la oclusión, y se observo la falta de guia en movimiento protrusivo y laterotrusivo la cual fue restituida. Finalmente, se le coloco una férula de relajación y protección.

\section{Referencia}

1. Tjan AH, Miller GD, The JD. Some esthetic factors in a smile. J Prosthet Dent. Jan 1984; 51(1):24-8. Doi: 10.1016/s0022-3913(84)80097-9

2. Garber DA, Salama MA. The aesthetic smile: diagnosis and treatment. Periodontol 2000. Jun 1996; 11:18-28. D o i : $10.1111 / \mathrm{j} .1600-$ 0757.1996.tb00179.x.

3. Silberberg N, Goldstein M, Smidt A. Excessive gingival display etiology, diagnosis, and treatment modalities. Quintessence Int. Nov-dic 2009; 40(10): 
809-18. PMID: 19898712.

4. Montalvo-Arias D, Molina G, Apa M. The "Gummy Smile" CHALLENGE: A Novel Multidisciplinary Approach. Journal of Cosmetic Dentistry. 2017; 33(2):50-61.

5. Trushkowsky R, Montalvo-Arias D, David S. Digital Smile Design concept delineates the final potential result of crown lengthening and porcelain veneers to correct a gummy smile. Int $\mathrm{J}$ Esthet Dent. 2016;11(3):338-54. PMID: 27433549.

6. Ribeiro-Júnior $\mathrm{NV}$, Campos $\mathrm{T}$, Rodrigues JG, Martins TM, Silva CO. Treatment of excessive gingival display using a modified lip repositioning technique. Int J Periodontics Restorative Dent. May-Jun 2013; 33 (3): 309-14. Doi: $10.11607 / p r d .1325$.

7. Gabric Panduric D, Blaskovic $M$, Brozovic J, Susic M, Surgical treatment of excessive gingival display using lip repositioning technique and laser gingivectomy as an alternative to orthognathic surgery. J Oral Maxillofac Surg. Feb 2014;72(2):404.e1-11. Doi: 10.1016/j.joms.2013.10.016.

8. Coachman C, Calamita M. Digital smile design: a tool for treatment planning and communication in esthetic dentistry quintessence of dental technology. Quintessence of Dental Technology (QDT). 2012; 35(103)1-10. Disponible en: shorturl.at/hMR17

9. Goyal M K, Goyal S,Hegde V, Balkrishana D, Narayana Al. Recreating an esthetically and functionally acceptable dentition: a multidisciplinary approach. Int J Periodontics Restorative Dent. Jul-agto 2013; 33(4): 527-32. Doi: 10.11607/prd.0381.

10. P. Magne, Hanna J, Magne. The case for moderate 'guided prep' indirect porcelain veneers in the anterior den- tition. The pendulum of porcelain veneer preparations: from almost no-prep to over-prep to no-prep. Eur J Esthet Dent. 2013; 8(3):376-88. PMID: 23957038.

11. Azer SS, Rosenstiel SF, Seghi RR, Johnston WM. Effect of substrate shades on the color of ceramic laminate veneers. J Prosthet Dent. Sept $2011 ; 106$ (3):179-83. Doi : 10.1016/S0022-3913(11)60117-0.

12. Gomez Henriques P. En Estética em Periodontia e cirurgia plástica periodontal. 2nd ed. São Paulo: Santos; 2003. ISBN: 85-7288-495-5

13. Brea L, Oquendo A, David S. Dental crowding: the restorative approach. Dent Clin North Am. Abr 2011; 55(2): $301-10, \quad$ i x . D o i : 10.1016/j.cden.2011.01.010.

14. Robbins JW. Differential diagnosis and treatment of excess gingival display. Pract Periodontics Aesthet Dent. Mar 1999;11 (2): 265-72; quiz 273. PMID: 10321231.

15. Ribeiro FV, Hirata DY, Reis AF, Santos VR, Miranda TS , Faveri M , Duarte PM Open-flap versus flapless esthetic crown lengthening: 12-month clinical outcomes of a randomized controlled clinical trial. J Periodontol. Abr 2014; $85(4): 536-44$. D o i : 10.1902/jop.2013.130145.

16. Arora R1, Narula SC, Sharma RK, Tewari S. Evaluation of supracrestal gingival tissue after surgical crownlengthening: a 6-month clinical study. J Periodontol. Jul 2013; $84(7): 934-40$. D o i : 10.1902/jop.2012.120162.

17. Brea L, Oquendo A, David S. Dental crowding: the restorative approach. Dent Clin North Am. 2011 Apr; 55 ( 2 ): $301-10$, ix. D o i : 10.1016/j.cden.2011.01.010.
Correspondencia:

drmachaca.proimplant@gmail.com
Fecha de recepción : :08 de julio de 2020

Fecha de aceptación : 22 de agosto de 2020 